\title{
Evidence for normal letter-sound integration, but altered language pathways in a case of recovered Landau-Kleffner Syndrome
}

Citation for published version (APA):

Pullens, P., Pullens, W., Blau, V., Sorger, B., Jansma, B. M., \& Goebel, R. (2015). Evidence for normal letter-sound integration, but altered language pathways in a case of recovered Landau-Kleffner Syndrome. Brain and Cognition, 99, 32-45. https://doi.org/10.1016/j.bandc.2015.07.003

Document status and date:

Published: 01/10/2015

DOI:

10.1016/j.bandc.2015.07.003

Document Version:

Publisher's PDF, also known as Version of record

Document license:

Taverne

Please check the document version of this publication:

- A submitted manuscript is the version of the article upon submission and before peer-review. There can be important differences between the submitted version and the official published version of record.

People interested in the research are advised to contact the author for the final version of the publication, or visit the DOI to the publisher's website.

- The final author version and the galley proof are versions of the publication after peer review.

- The final published version features the final layout of the paper including the volume, issue and page numbers.

Link to publication

\footnotetext{
General rights rights.

- You may freely distribute the URL identifying the publication in the public portal. please follow below link for the End User Agreement:

www.umlib.nl/taverne-license

Take down policy

If you believe that this document breaches copyright please contact us at:

repository@maastrichtuniversity.nl

providing details and we will investigate your claim.
}

Copyright and moral rights for the publications made accessible in the public portal are retained by the authors and/or other copyright owners and it is a condition of accessing publications that users recognise and abide by the legal requirements associated with these

- Users may download and print one copy of any publication from the public portal for the purpose of private study or research.

- You may not further distribute the material or use it for any profit-making activity or commercial gain

If the publication is distributed under the terms of Article 25fa of the Dutch Copyright Act, indicated by the "Taverne" license above, 


\title{
Evidence for normal letter-sound integration, but altered language pathways in a case of recovered Landau-Kleffner Syndrome
}

\author{
Pim Pullens ${ }^{\mathrm{a}, \mathrm{b}, *}$, Will Pullens ${ }^{\mathrm{c}}$, Vera Blau ${ }^{\mathrm{a}}$, Bettina Sorger ${ }^{\mathrm{a}}$, Bernadette M. Jansma ${ }^{\mathrm{a}}$, Rainer Goebel ${ }^{\mathrm{a}, \mathrm{b}}$ \\ ${ }^{a}$ Maastricht University, Maastricht Brain Imaging Center, Faculty of Psychology and Neuroscience, Oxfordlaan 55, 6229 EV Maastricht, Netherlands \\ ${ }^{\mathrm{b}}$ Brain Innovation BV, Research \&' Development, Oxfordlaan 55, 6229 EV Maastricht, Netherlands \\ ${ }^{\text {c } R o y a l ~ D u t c h ~ K e n t a l i s, ~ S c h o o l ~ M a r i e ̈ l l a, ~ T h e e r e s t r a a t ~ 42, ~} 5271$ GD Sint Michielsgestel, Netherlands
}

\section{A R T I C L E I N F O}

\section{Article history:}

Received 26 September 2014

Revised 2 March 2015

Accepted 14 July 2015

Available online 29 July 2015

\section{Keywords:}

Landau-Kleffner Syndrome

fMRI

DTI

Arcuate fasciculus

Auditory cortex

Audiovisual

Multisensory integration

\begin{abstract}
A B S T R A C T
Landau-Kleffner Syndrome (LKS) is a rare form of acquired aphasia in children, characterized by epileptic discharges, which occur mostly during sleep. After normal speech and language development, aphasia develops between the ages of 3-7 years in a period ranging from days to months. The epileptic discharges usually disappear after reaching adulthood, but language outcomes are usually poor if no treatment focused on restoration of (non-) verbal communication is given. Patients often appear deaf-mute, but sign language, as part of the treatment, may lead to recovery of communication. The neural mechanisms underlying poor language outcomes in LKS are not yet understood. In this detailed functional MRI study of a recovered LKS patient - that is, a patient no longer suffering from epileptic discharges, audiovisual multi-sensory processing was investigated, since LKS patients are often proficient in reading, but not in speech perception. In the recovered LKS patient a large difference in the neural activation to auditory stimuli was found in the left versus the right auditory cortex, which cannot be attributed to hearing loss. Compared to healthy proficient readers investigated earlier with the same fMRI experiment, the patient demonstrated normal letter-sound integration in the superior temporal gyrus as demonstrated by the multi-sensory interaction index, indicating intact STG function. Diffusion Tensor Imaging (DTI) based fiber tracking in the LKS patient showed fibers originating from Heschl's gyrus that seem to be left-right inverted with respect to HG fiber pattern described in the literature for healthy controls. In the patient, in both hemispheres we found arcuate fibers projecting from (homologues of) Broca's to Wernicke's areas, and a lack of fibers from arcuate left inferior parietal and sylvian areas reported in healthy subjects. We observed short arcuate segments in the right hemisphere. Although speculative, our results suggest intact temporal lobe processing but an altered temporal to frontal connectivity. The altered connectivity might explain observed short-term verbal memory problems, disturbed (speech) sound-motor interaction and online feedback of speech and might be one of the neuronal factors underlying LKS.
\end{abstract}

(c) 2015 Elsevier Inc. All rights reserved.

\section{Introduction}

Landau-Kleffner Syndrome (LKS) was first described in 1957 (Landau \& Kleffner, 1957) as a "form of acquired auditory aphasia in children", and it is characterized by either partial or total loss of auditory comprehension or inability of environmental sound discrimination (Steinlein, 2009). Together with aphasia symptoms of epilepsy start to occur. Patients suffer from epileptic discharges occurring in both temporal regions, which become almost continuous during slow-wave sleep (Steinlein, 2009), and LKS is thus

\footnotetext{
* Corresponding author at: Antwerp University Hospital \& University of Antwerp, Department of Radiology, Wilrijkstraat 10, 2650 Edegem, Belgium.

E-mail address: pim.pullens@uantwerpen.be (P. Pullens).
}

considered as a subtype of continuous slow waves during sleep (CSWS). Most epileptic symptoms tend to disappear when the patients enter adolescence.

When symptoms of LKS first arise, the patient seems to be deaf, but audiograms are usually normal (Feekery, Parryfielder, \& Hopkins, 1993). The cause of LKS, its pathophysiology and the neural mechanisms behind the language disorders in LKS are not yet understood (Fandiño, Connolly, Usher, Palm, \& Kozak, 2011; Hirsch et al., 2006).

LKS children initially acquire speech and language in a normal way. At the age of three to seven years, they develop (semi-) acute aphasia in a period of days to months. Aphasia is mostly receptive (phonological decoding) and develops into expressive impairments at a later stage (Lanzi, Veggiotti, Conte, Partesana, \& Resi, 1994). 
Auditory agnosia, i.e. the inability to distinguish sounds is also reported in LKS. Currently the language disorders in LKS are therefore classified as verbal agnosia or auditory agnosia (Cockerell, Bølling, \& Nakken, 2011). It is unclear whether the inability to distinguish sounds reflects a specific phonological decoding deficiency or whether there is a more general auditory-perceptual processing deficit affecting the analysis of any type of sounds (verbal or non-verbal) (Pedro \& Leisman, 2005).

As well as language disorders other higher-order cognitive functions such as memory and attention can be affected as well. These deficits may arise as a consequence of epileptic seizures and/or their associated treatment. These cognitive dysfunctions are often associated with dramatic changes on a behavioral level. Two-thirds of studied LKS patients show signs of behavioral change including aggression, attention and concentration disorders, as well as resistance against behavioral change (disengagement, task switching), echolalia, echopraxia, and even psychotic behaviors (Zivi, Broussaud, Daymas, Hazard, \& Sicard, 1990). This might also be correlated to an inability to link sounds to facial expressions and/or body language. Normally, humans are very good in connecting emotional sounds to facial expressions (i.e. sound of laughter with a smiling face (De Gelder \& Vroomen, 2000) or in associating body language with emotional sounds (Van den Stock, Grèzes, \& de Gelder, 2008)), but if this connection is lost, emotional sounds will not be interpreted correctly and will likely lead to fear in the patient.

Behavioral treatment of any form of audio-visual communication usually leads to a reduction of problematic behavior (Appleton, 1995; Deonna, Prelaz-Girod, Mayor-Dubois, \& Roulet-Perez, 2009) and quality of life is greatly improved. The degree of recovery is determined by the time of onset, the response to anti-epileptic medication and the severity of communication problems. To our knowledge there are no reports of full recovery and individual variation is high; while some patients may have permanent language disorder, some regain much of their language capabilities.

Neuro-anatomical abnormalities in LKS patients have been reported based on volumetric MRI analysis. These abnormalities are mainly gray matter volume reduction in bilateral superior temporal areas, foremost in planum temporale and superior temporal gyrus (Takeoka et al., 2004).

This anatomical finding received support by functional studies. Dysfunction of superior-temporal and perisylvian areas, both during the active phase as well as long lasting dysfunction was reported using a single or four word repetition task during positron emission tomography (PET) (Majerus et al., 2003). The tip of the left temporal lobe was also found to be inactive in a LKS case study using resting-state PET (Shiraishi, Takano, Shiga, Okajima, \& Sudo, 2007).

Electroencephalography (EEG) studies during the active epileptic phase have shown that auditory information (speech and natural sounds) seems to be processed normally within the brain stem, as the EEG is normal for the early (brain stem related) auditory processing time window (Steinlein, 2009).

With regard to more complex auditory integration, LKS patients in the active and recovered phase suffer from permanent dysfunction of associative auditory cortex as measured in a dichotic listening task during EEG recordings (Metz-Lutz, de Saint Martin, Hirsch, Maquet, \& Marescaux, 1999; Plaza, Rigoard, Chevrie-Muller, Cohen, \& Picard, 2001; Wioland, Rudolf, \& Metz-lutz, 2001). This dysfunction is expressed as unilateral dichotic extinction, contra-lateral to the epileptic focus (Wioland et al., 2001). Furthermore, short-term phonological memory is impaired in LKS, even in patients who have recovered fairly well (Majerus et al., 2003).

It seems that language functions in LKS are reorganized from the left to the right hemisphere, as was demonstrated in a single case follow-up fMRI study (Datta et al., 2013). The initial active epileptic focus for this patient was localized with source EEG in the left fronto-centro-temporal area. The language network detected by fMRI was also left lateralized initially, but seems to have transferred into the right hemisphere as observed by follow-up fMRI.

There is no impairment in the visual domain in LKS; one important observation in the clinics in contact with LKS patients is that many of them show astonishing recovery of communication skills using alternate means, such as non-verbal signing (Deonna et al., 2009; Perez et al., 2001; Stefanatos, 2011). Moreover, the ability to learn and use written language is found not to be impaired (Denes, 1998). The patient described in the latter study had "a flawless performance in lexical decision tasks, as well in the written naming subtest of BDAE (..) and in written naming following semantic cuing" (Denes, 1998).

The question we address here is what is the neural mechanism that allows LKS patients to compensate for their sudden loss of auditory language functions? If the typical route to access meaning about objects/events via spoken language is blocked, how does the brain accommodate the plastic changes needed to recover communication skills?

The present study simultaneously examined auditory, visual and audiovisual processing abilities in LKS using the smallest possible units of spoken and written language (speech sounds and letters). When healthy children learn how to read, a crucial first step is to learn the correspondence between written letters and speech sounds (Van Atteveldt, Formisano, Goebel, \& Blomert, 2004). This integration of written letters and letter sounds (multimodal integration) is acquired with high efficiency in a normal developing child (Van Atteveldt et al., 2004) but may be impaired in LKS.

The goal of the present investigation was twofold. Firstly, we aimed to examine the unimodal (only written letters/only letter sounds) and multimodal (simultaneous written letters and letter sounds) integration of letters and sounds using functional magnetic resonance imaging (fMRI). We studied the case of an adult female LKS patient whose communication abilities were largely restored following long-term multi-sensory intervention training (see case description). We compared the results of uni- and multimodal processing found in the patient with the results from an identical experiment in healthy controls $(N=12)$ (Blau, Van Atteveldt, Ekkebus, Goebel, \& Blomert, 2009). Second, the language disorders might be related to abnormalities in connectivity of the temporal and associative cortices in the epileptic phase (Hirsch et al., 2006), therefore a second major goal of this paper is to investigate the white matter pathways in temporal and associative cortex in a LKS patient after the active epileptic phase with DTI. Specifically, the connections arising from the primary auditory cortex were reconstructed to investigate how Heschl's gyrus is connected to higher language areas. In addition, we investigated the arcuate fasciculus, as this fiber bundle connects the most relevant functional areas of interest related to language and information integration between temporal lobe and frontal lobe. Specifically we looked into connections between inferior frontal gyrus and superior temporal gyrus/superior temporal sulcus.

\section{Materials and methods}

\subsection{Subjects}

\subsubsection{Recovered LKS patient}

We present a case of a 27-year old female "I.", diagnosed with LKS. At present she lives a full live as an educated married woman with a full time job. In the cause of this investigation she had an audiometric evaluation (Viataal Audiometric Centre NL 2009), 
her audiogram was found to be in a normal range (right and left ear $<20 \mathrm{~dB}$ in the $200 \mathrm{~Hz}-8 \mathrm{kHz}$ range). We are able to present a detailed case and development description since author WP has been working with I. on speech and language therapy from early childhood until the present.

She was invited to participate in the experiment, which was voluntary and in accordance with the Maastricht University ethical guidelines. Informed consent was obtained from the subject.

\subsubsection{Early childhood}

I. was born from a full-term gestation with uncomplicated pregnancy and delivery. First development was normal including normal language development. At the age of 4 , her parents and the preschool teacher observed problems in language comprehension. At the time, she was perceived to suffer from progressive hearing loss. Neurological examination (24 h EEG at the Sint Radboud Hospital Child Neurology Center, Nijmegen, NL) lead to the diagnosis of Landau-Kleffner Syndrome.

The awake EEG showed low-frequency peaks or sharp wave complexes, which were sometimes better expressed in the right than in the left hemisphere with topographic maxima in central/temporal regions. Bilateral bursts were also observed. During sleep, epileptiform activity was seen in $80 \%$ of the recorded EEG, and during Rapid Eye Movement (REM) sleep the epileptiform focus was right-central lateralized.

At the age of five, language comprehension and language production became increasingly disrupted. A year later, she was examined by a neuropsychologist, who did not observe any cognitive or visuo-motor impairments, however her speech-language disorders seemed to be caused by sensory aphasia. Additionally, language perception was severely disrupted on a word/sentence level and she was diagnosed with auditory agnosia and jargon-aphasia, where speech is incomprehensible but seems to make sense to the patient. Mixed-type aphasia, which is not solemnly caused by auditory agnosia, combined with a very weak auditory memory was diagnosed.

Expressive language was severely impaired as well as was the abstraction of inner language. Memory of rhythm was pathologically weak, as were fine motor skills and memory of order of motor tasks. Visual memory was intact; she had developed a "coding strategy", that is the use of non-verbal gestures, mime, and objects for communication. Lastly, non-verbal abstraction was weak and hence problems in relating written letters to letter sounds were to be expected.

At the age of seven, she scored in the average to above-average (IQ 115) on the executive part of an intelligence test (Son-r non-verbal intelligence test, www.testresearch.nl). Performance on visual memory was equal or above average compared to her age group. Spontaneous speech was characterized by jargon-aphasia. Some meaningful two- and multiple-word sentences were produced. At the articulation test, almost all words were correctly pronounced. Errors consisted of word reduction. However, she was severely impaired on auditory discrimination and, comprehension of sentence structure, fluency of speech and auditory memory.

At this point she was communicative, and focused on body language and facial expressions of the other party to get additional information. However, it was not to be expected at this point in time that oral communication would be possible. Results indicated positive skills with respect to inner language, concept forming and using pictograms. Based on these investigations, she was admitted into a specialized school for the deaf. The school curriculum, developed for deaf children with learning disabilities is based on visualized and written conversation and the use of finger spelling. The finger spelling system is the American Manual Alphabet used in American Sign Language.
In the first evaluation, the Kaufmann Intelligence Test (Kaufman \& Kaufman, 1985; Mulder, Dekker, \& Dekker, 1993) indicated that she fitted the profile of the school's pupils. The Kaufman intelligence test (Dutch/Flemish edition) measures intelligence in different domains. The average reference score on each subset of the test, adjusted for The Netherlands and Flanders is 10 and standard deviation is 3 . The standardised scores of I. on hand-movement: 9, number recall: above average, face recognition: average, gestalt closure: 8 , triangles: 14 , matrices: 16 , spatial memory: 10 , photo series: 14. The total score was above 91, indicating average intelligence.

Her learning conditions for oral language, however, were so weak that communication by speech was not possible during this time. The ability to process information that is spatial in nature and presented simultaneously was strong and written language was predicted to play an important role in language development. The ability to process temporally ordered information was weak, causing problems when reading finger spelled language. However, after an introduction period, active use of fingerspelling was possible. Understanding spoken language was poor; almost no words were being recognized based on hearing only.

\subsubsection{Education}

At the age of 13 she started secondary schooling at a school for children with specific language impairment (SLI). After an intermediate year in practice-based education she reached a level sufficient to start high school. Hereafter she was educated as a teaching assistant to a Dutch MBO level (comparable US Associate Degree) in a regular institute. She passed the theoretical exams without problems given that reading materials were available. Oral instructions/teaching were only possible when the teacher made eye contact with her. It turned out to be impossible for her to do a standard internship as teaching assistant in a classroom, since she could only understand children when they made eye contact. In a group, she was found to be unable to understand a child and to select a single voice in a group. Therefore, she did an internship in a school for children with severe communication disorders, which she completed successfully. Recently (2009), she was awarded a bachelor's degree as a teaching assistant which she obtained in the standard time period.

\subsubsection{Present}

At present, she has a full-time job as a teaching assistant at a school for the deaf. In order to fully understand colleagues and children, she still needs to make eye contact. This also means that I. cannot use a telephone. She continues to make mistakes when speaking, in word form, sentence construction as well as melody and accent. During work-related meetings, a strict communication policy is used: each speaker has to indicate when he/she is speaking and speakers have to speak in concession, not simultaneously. In bigger meetings, a writing interpreter is used in order to help her understand the Dutch language in full.

School practitioners developed the hypothesis that her language disorder can be overruled by using the motor system (finger spelling and gestures) instead of verbal auditory input, which is in agreement with positive results obtained when using finger spelling in LKS (Deonna et al., 2009; Stefanatos, 2011). In this way spoken language skills were partly restored and understanding of spoken language based on lip reading and gestures became possible. In adulthood all former students who had followed a similar program, expressed motor involvement - visible hand twitches - while understanding spoken or written language.

Previous single case evaluations indicated the importance of alternative visual communication systems in order to prevent behavioral problems, emotional disturbances and delayed cognitive development (Deonna et al., 2009). It was stated that the use 
of sign language or signed oral language, including finger spelling, enabled successful recovery of communication functions. The following suggestion was made: "Important issues like successful delayed restoration of unused cortical functions or the occasional occurrence of good reading without phonology or the reorganization of brain functions should be studied in patients with LKS" (Deonna et al., 2009).

\subsubsection{Control subjects}

We compared the patient data with the original MRI data of healthy controls (Blau et al., 2009), with permission. MRI data on 12 healthy subjects, 4 females, mean (std) age 22.8(3.8) years were acquired using the identical experimental paradigm and data acquisition protocol on the same MRI scanner as for the patient data.

\subsection{MR imaging}

\subsubsection{Functional magnetic resonance imaging}

The stimuli and presentation design were adapted from Blau and colleagues (Blau et al., 2009). Stimuli were visual letters and auditory speech sounds corresponding to Dutch single letters. Presented consonants were: $b, d, g, h, k, l, n, p, r, s, t, z$; vowels: a, e, i, y, o, u. Stimuli were presented with Presentation software (Neurobehavioral Systems, Inc.) in blocks corresponding to four experimental conditions: unisensory letters (L): written "a", unisensory speech sounds (S): spoken "/a/", multisensory congruent LS pairs "a" "/a/", and multisensory incongruent LS pairs "a" "/b/". One block was $20.8 \mathrm{~s}$ long, divided into four mini-blocks (5.2 s). Each mini-block is composed of the MRI acquisition followed by five silent stimuli presentations. During multisensory stimulation, stimuli were presented simultaneously. Subject passively listened to and/or viewed the stimuli. The experiment included four experimental runs, each composed of eight blocks and alternating fixation periods. Each condition (40 trials) was repeated twice per run. The order of blocks was pseudo-randomised within runs, and the order of runs was counterbalanced across subjects.

The experiment was explained and guided by her former teacher and present colleague using speech and finger spelling, to make sure that there were no communication problems while instructing the subject.

MR imaging was performed on a 3T Allegra MR scanner (Siemens, Erlangen, Germany) using a birdcage single coil. Blood Oxygenation Level-Dependent (BOLD) signal was acquired with a $\mathrm{T} 2 *$ sensitive Echo-Planar Imaging (EPI) sequence and $\mathrm{TR} / \mathrm{TE}=5200 \mathrm{~ms} / 30 \mathrm{~ms}$. We acquired $244.5 \mathrm{~mm}$ thick slices covering the whole brain, in a $64 \times 64$ matrix with Field of View (FOV) $192 \times 192$, resulting in $3 \times 3 \times 4.5 \mathrm{~mm}$ voxels. The volume acquisition time was $1500 \mathrm{~ms}$, followed by a silent period of $3700 \mathrm{~ms}$, during which stimuli were presented. Additionally, a high quality T1 weighted anatomical image was acquired using an ADNI MP-RAGE sequence (TR/TE 2250/2.6 ms) with 192 slices and a matrix of $256 \times 256$. The FOV was $256 \times 256 \mathrm{~mm}$, resulting in $1 \times 1 \times 1 \mathrm{~mm}$ voxels.

Data analysis was performed in BrainVoyagerQX 2.1 (Brain Innovation BV, Maastricht, NL). Functional data were first corrected for slice scan time differences, subject motion artifacts, linear drifts and low-frequency drifts using default settings. The fMRI data were then co-registered to the anatomical data and converted into standard Talairach space. Due to severe artifacts, the third run had to be discarded from the fMRI data set.

The fMRI runs were analyzed using voxel-wise multiple linear regression (General Linear Model GLM) of the BOLD time course. The predictors - or regressors - for the GLM were modeled as a convolution of the stimulus box-car time course (one for each stimulus condition "Letters", "Sounds", "incongruent L/S pairs", "congruent L/S pairs") with a standard hemodynamic response function (HRF), the 2-Gamma HRF. The 3 available runs were fed into a fixed-effects GLM with $z$-normalization of the time courses of each run to produce $t$-statistic maps. The maps were thresholded at $t=2.839$ with 197 degrees of freedom, resulting in $p=.005$.

To correct for multiple comparisons, we estimated a cluster threshold according to the method described in Goebel, Esposito, and Formisano (2006) at a p-value of 0.005 , which reduces the probability of type II errors commonly found when using the False Discovery Rate (Lieberman \& Cunningham, 2009). The cluster threshold was estimated at 3 voxels $\left(81 \mathrm{~mm}^{3}\right)$. Percent BOLD signal change was computed using a GLM with percent transform. Each value in the time course is divided by the mean of the time course and then multiplied by 100 , thus obtaining a percent signal change (BrainVoyagerQX User Guide). The extracted BOLD response in selected Regions of Interest (ROIs) was further processed in Matlab R2007b (The Mathworks, USA) using the BVQXtools toolbox, available on http://support.brainvoyager.com/availabletools/52. Because of non-normality of the data (tested with a probability plot using the "normplot" function in Matlab), statistical tests on the \% BOLD signal change were done by a Wilcoxon rank sum test for equal medians, with $p=0.05$.

From the Talairach normalized anatomical data, cortical reconstructions were created using an automatic cortex reconstruction procedure. The GLM results were projected on the 3D cortical representation sheet and ROIs were defined on the cortical sheet. Talairach coordinates of significantly active regions were visually inspected and subsequently verified using the Talairach daemon (http://www.talairach.org/).

In the control group 4 functional runs were obtained per subject. To obtain comparable statistical power in the control and patient data, we discarded one randomly selected functional run from each control subject data set. The data per subject was analyzed identically to the patient data.

We specifically investigated the volume of significantly activated regions to the unisensory auditory stimulus in the left and right primary auditory cortex. The volume of the response areas was obtained by determining activation cluster size with a threshold of 300 anatomical voxels in the primary auditory cortex. The volume fraction of the response area was calculated as

volume fraction $_{\mathrm{L}, \mathrm{R}}=\frac{V_{\mathrm{AUD}}}{V_{\mathrm{AUD}, \mathrm{L}}+V_{\mathrm{AUD}, \mathrm{R}}} \cdot 100$

where $V_{\mathrm{AUD}, \mathrm{LR}}$ are the volumes of the left and right primary auditory responses in $\mathrm{mm}^{3}$ and $V_{\mathrm{AUD}}$ is the volume of the right or the left response area.

Statistically robust across-subject activation patterns in the control group were created by analyzing the data in a random effects (RFX) GLM approach with percent transformation. Because of the unbalanced group size of 12 controls and 1 patient, we applied an analysis similar to Cavina-Pratesi, Kentridge, Heywood, and Milner (2009). To allow for direct comparison with the patient data, the group RFX-GLM results and the patient results were thresholded at $t=3.428$ resulting in $p=0.005$. The optimal cluster threshold was calculated using Monte Carlo simulations in BrainVoyagerQX. The cluster size threshold was $20 \mathrm{fMRI}$ voxels $\left(540 \mathrm{~mm}^{3}\right)$ for the group and $3 \mathrm{fMRI}$ voxels $\left(81 \mathrm{~mm}^{3}\right)$ for the patient.

\subsubsection{Diffusion tensor imaging}

White matter structure can be investigated non-invasively with diffusion-weighted MRI. By probing for microscopic water diffusion in white matter axons with MRI and following the main direction of the diffusion tensor (diffusion-tensor based fiber tracking 
"DTI-based fiber tracking"), this technique allows for reconstruction of WM fiber tracts in the brain (for an overview of the technique, see e.g. (Mori \& Van Zijl, 2002) DW-MRI data were acquired in the same recording session as the functional data. A double-refocussed diffusion weighted SE-EPI sequence was used to optimize data quality by minimizing the effects of eddy current distortions. 75 slices (thickness $2 \mathrm{~mm}$ ) covering the whole brain were acquired with matrix size $128 \times 128$, field of view $256 \times 256$, resulting in $2 \times 2 \times 2 \mathrm{~mm}$ voxels. 72 isotropically distributed diffusion directions at a $b$-value of $1000 \mathrm{~mm}^{2} / \mathrm{s}$ were acquired, as well as 7 interleaved b0 volumes. TR/TE 9400/83 ms, acquisition time was $12 \min 53 \mathrm{~s}$.

\subsubsection{DW-MRI data processing}

The DWI data was checked for artefacts related to motion by an affine registration of the non-diffusion weighted $b 0$ volumes. No significant motion was detected (the registration error was well below 1 voxel), so no motion correction was performed. The data was checked for eddy current distortions using a modified version of the ICC algorithm (Zhuang et al., 2006). Very little distortion was found due to the double-refocussed SE-EPI sequence and therefore eddy current correction was omitted.

The DWI data was co-registered to the anatomical data set in BrainVoyagerQX 2.2 (Brain Innovation BV, Maastricht NL). The diffusion tensors were calculated in BrainVoyagerQX and exported to in-house developed $\mathrm{C} / \mathrm{C}++$ fiber tracking software. For fiber tracking, an FA threshold of 0.15 was set. The angular threshold was set at 30 degrees and the step size at $0.5 \mathrm{~mm}$. Reconstructed fibers were visualized in BrainVoyagerQX on the anatomical data set. Cross-validation of the AF fiber tracts was performed in ExploreDTI (Leemans, Jeurissen, Sijbers, \& Jones, 2009). Constrained Spherical Deconvolution (CSD) reconstruction and fiber tracking were done using default settings.

From the anatomical data, the gray matter cortical sheet was reconstructed in BrainVoyagerQX. On the cortical sheet, Heschls gyrus was marked as a 3-D ROI in accordance with the delineation in Sigalovsky, Fischl, and Melcher (2006). The ROI was then back-projected onto the DWI data set in order to create a 2-D ROI. The ROIs needed to reconstruct the arcuate fasciculus were created on axial slices of the DW-MRI images according to the ROI definitions in Catani et al. (2007), Gharabaghi et al. (2009).

For comparison of the reconstructed fiber tracts in this single case to control data, we used the arcuate fibers supplied in the white matter atlas from Thiebaut de Schotten et al. (2011), which is composed of major fiber tracts in 40 right-handed healthy control subjects (20 male and 20 female) aged 18-22 years. The anatomical image was co-registered to the average atlas brain anatomy using FSL-FLIRT (Smith et al., 2004).

The reconstructed AF fibers were back-projected on the subjects anatomical image, and then converted to Nifti using the Nifti-converter v1.08 for BrainVoyagerQX. The registration matrix obtained from FSL-FLIRT was used to convert the reconstructed fibers of the arcuate fasciculus in the patient into the atlas brain space.

\section{Theory}

In order to understand the neural mechanisms underlying the auditory and language dysfunctions in LKS, we reviewed the extensive literature on brain language networks. The recent "dual-stream" model (Hickok \& Poeppel, 2007) for speech processing is one of the most advanced models for speech processing to date. The model, summarized in Fig. 1, suggests that sensorimotor processes are key in speech perception. Early speech processing is done in the superior temporal gyrus and sulcus (STG/STS). The STG performs spectrotemporal analysis of the input signal, and STS phonological access and representation. Further processing occurs in two streams: a temporal lobe ventral stream for speech comprehension (lexical access and combinatorial processes) and a strongly left dominant stream for sensorimotor integration (Hickok, Houde, \& Rong, 2011).

The motor speech system is activated during passive listening to speech and may provide a modulatory influence on speech perception (Hickok et al., 2011). The responsible neural network is comprised of premotor cortex, area Spt, STG and the cerebellum. After passing through this network, speech input is passed through to higher areas for lexical and conceptual processing.

Normally, literacy is acquired later in development than spoken language and takes more effort than spoken language. Spoken language is more natural and acquired without effort. In healthy normal readers, skilled reading is very likely to still automatically activate phonological representations (Van Atteveldt, Roebroeck, \& Goebel, 2009).

The model for integration of letters and sounds adds visual input to the system. Visual and auditory input is combined in the STG and then processed further on in the system along the
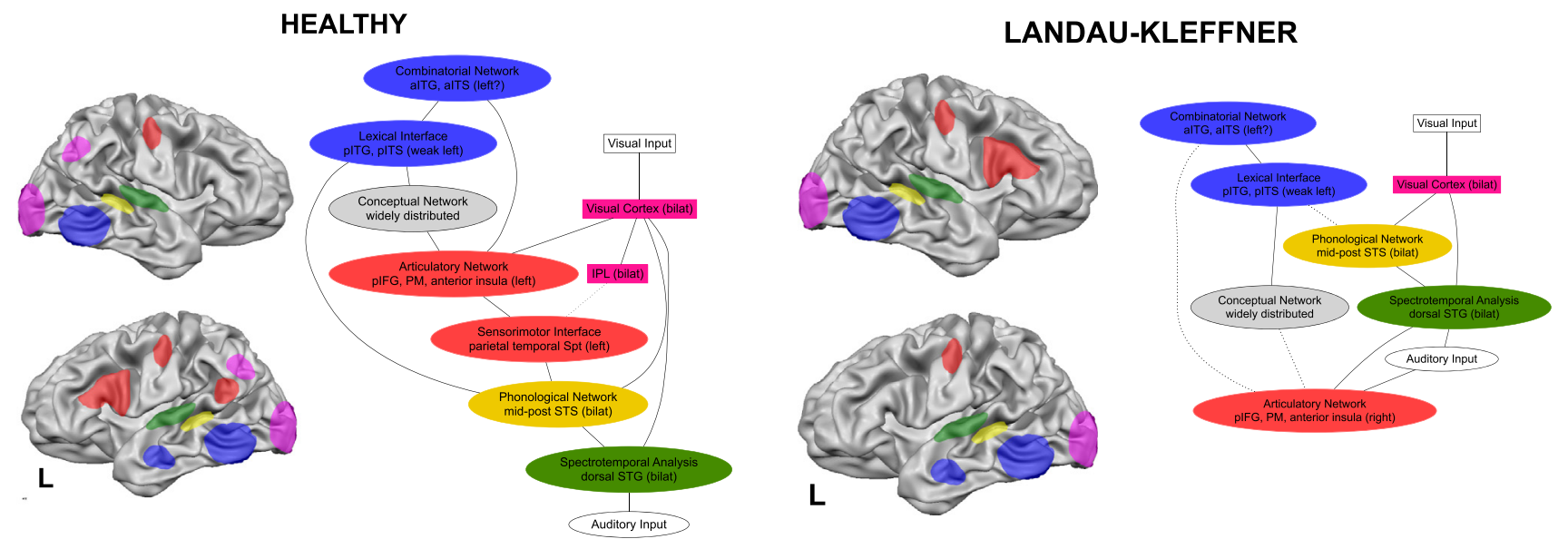

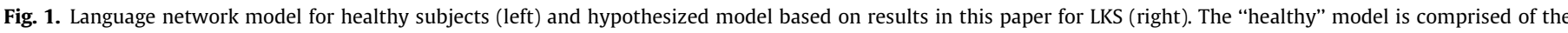

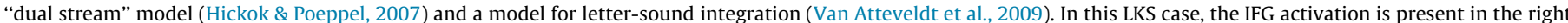

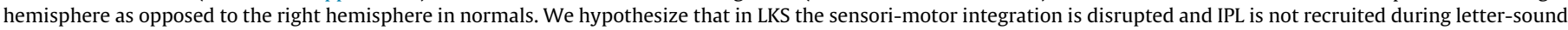
integration (see text for further explanation). 
fasciculus (Van Atteveldt et al., 2009). From the STG, there is also feedback to the auditory cortex if stimuli are presented synchronously. Secondly, there might be feedback to the visual cortex from the STG. The authors do not discuss the role of the inferior parietal lobule (IPL) in this network, however there is evidence from DTI-based fiber tracking that IPL is part of the dorsal stream (Catani, Jones, \& fFytche, 2005).

Taken together the model of speech processing and findings from PET/EEG suggest that a failure to transmit incoming auditory information beyond early sensory cortices in the temporal lobe leaves LKS patients unable to access meaning from spoken words, as the processing within the language network within temporal lobe is hampered due to limited connectivity in this region.

\section{Results}

\subsection{Functional MRI}

\subsubsection{Response to unisensory stimuli}

To locate the regions where the unisensory stimuli differ significantly, the contrast auditory > visual was set (contrast vector [auditory visual congruent incongruent $]=[1-100]$ ). Fig. 2 shows the brain regions in which neural responses were driven by speech sounds as opposed to visual letters as a $t$-statistic map with $p<0.005$ and a cluster size threshold of 3 fMRI voxels $\left(81 \mathrm{~mm}^{3}\right)$. A more detailed overview of the brain regions is given in Table 1. Regions activated by the auditory stimulus include the primary auditory cortex (PT/HG BA 41/42) bilaterally, which shows a remarkable difference in the activation pattern between the right and left hemisphere. The activation in the left hemisphere shows a patch-like pattern, while the activation in the right hemisphere shows a more equally distributed pattern. The size of the right hemispheric activation is significantly larger than the left hemispheric region, see Table 1 and Fig. 2 . In the healthy subjects the left and right primary auditory cortex activations are similar in size, see Fig. 3. The average volume fraction of the left primary auditory cortex is $53.7 \%$ and right is $46.3 \%$ in the controls, while it is $17.5 \%$ versus $82.5 \%$ in the LKS patient. The volume fraction in the LKS patient is significantly different from the control group ( $t$-test, $t=5.310, p=2.4 \mathrm{e}-4$ ).

Additional regions preferentially activated by speech sounds versus visual letters include the superior temporal sulcus/gyrus (STS/STG, BA 41/42, BA 13) and pre-central gyrus (preCG, Brodmann Area BA 4) bilaterally. Remarkably, we found that similar regions in the controls respond to the visual stimuli, see Fig. $5 \mathrm{c}$ and $\mathrm{d}$.

In the left hemisphere a region in the precentral gyrus (BA 6) was also activated. Brain areas that preferentially responded to the presentation of visual letters as opposed to speech sounds include the primary visual cortex, lateral occipital regions BA $18 / 19$, as well as the fusiform gyrus (BA 37) bilaterally. In the left hemisphere, a region in the precentral gyrus (BA 6), and two regions in the precuneus (BA 7,31 ) were also active in response to the visual stimulus.

We investigated the hemodynamic response (BOLD response) in the primary auditory cortex more closely with a ROI analysis. The ROIs encompassed the primary auditory cortex BA 41, center of gravity $(x, y, z)=(42,-35,14)$ and $(-38,-35,14)$ and visual cortex BA $18,(x, y, z)=(26,-76,-8)$ and $(-35,-81,-8)$. Fig. 4 shows the BOLD response in primary auditory and visual cortex to the unimodal stimuli (only visual/only auditory). The primary auditory cortex shows a typical activation BOLD response to the auditory stimulus, while the BOLD response to the visual stimulus did not significantly differ from baseline. In the visual cortex, we observe a response to the visual stimulus, while the response to the auditory stimulus remains at baseline level. Combined with the results of the audiogram (normal range) it can be stated that this subject has the capacity to detect sounds, including vocals and language.

\subsubsection{Relative contribution of auditory and visual stimuli}

In Fig. 5, the relative contribution of auditory and visual stimuli is shown on the inflated cortical sheet. Relative contribution of the beta-values, with $v$ for visual and $a$ for auditory, is computed as

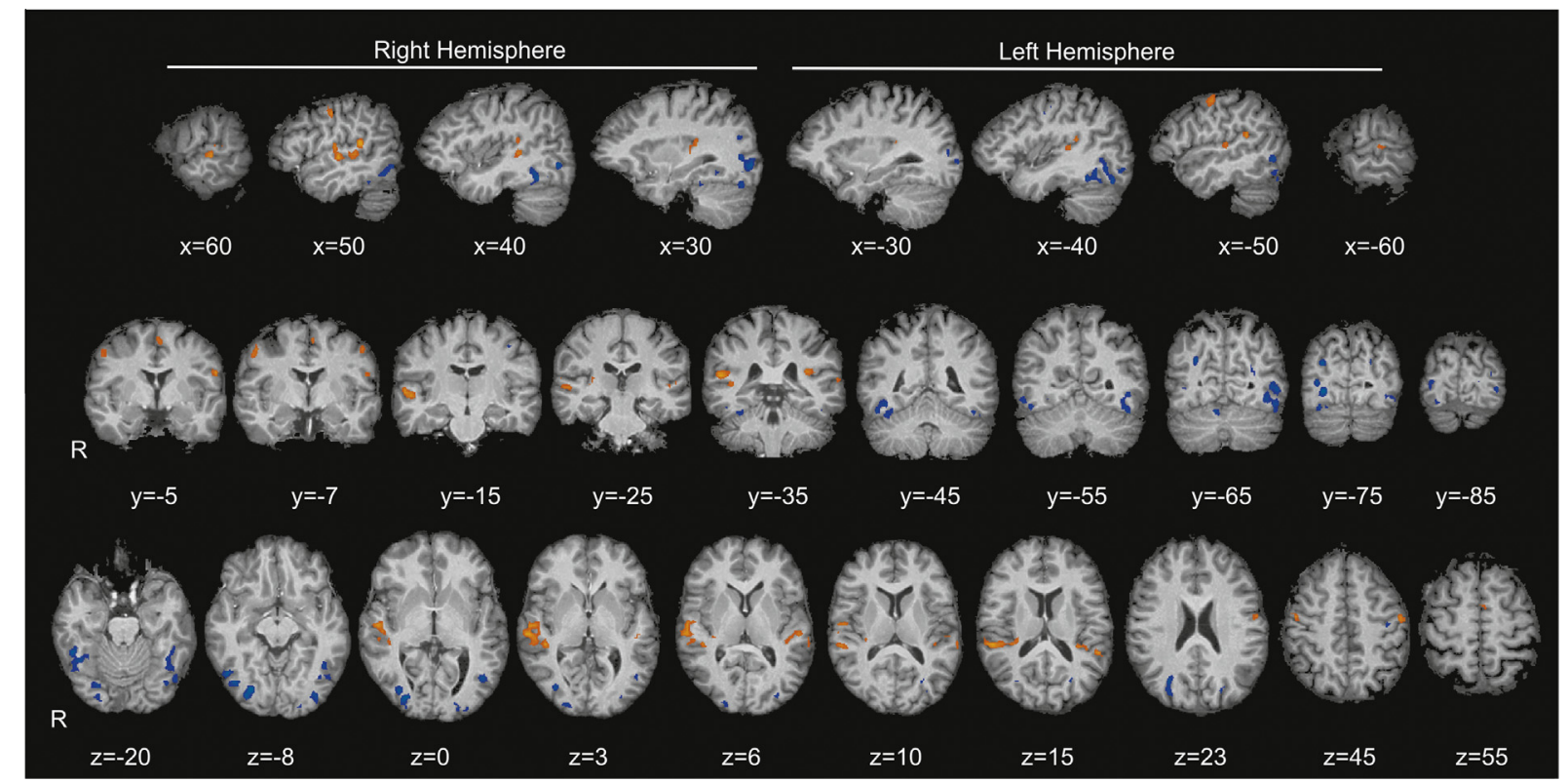

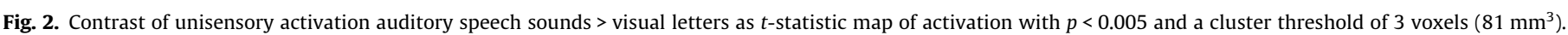

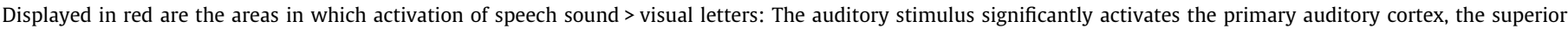

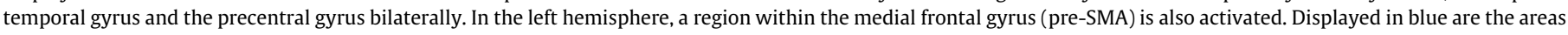

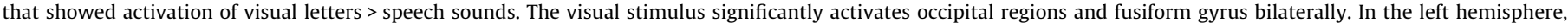

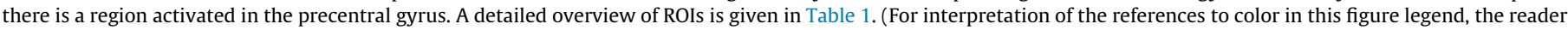
is referred to the web version of this article.) 
Table 1

Regions activated by the unisensory conditions with $p<0.005$ and cluster size 3 voxels in the LKS patient, and in the control group. Center of gravity coordinates for LKS only in Talairach coordinates $(\mathrm{mm})$ and size in $\mathrm{mm}^{3}$. BA: Brodmann Area; VIS: visual stimulus; AUD: auditory stimulus. Gray rows represent areas that are activated by a different stimulus in LKS versus controls.

\begin{tabular}{|c|c|c|c|c|c|c|c|c|}
\hline \multirow[b]{3}{*}{ Region } & \multicolumn{5}{|c|}{ Left Hemisphere } & \multirow[b]{3}{*}{ size } & \multirow{2}{*}{\multicolumn{2}{|c|}{ Controls }} \\
\hline & \multirow{2}{*}{\multicolumn{3}{|c|}{ Center of gravity }} & \multirow{2}{*}{$\begin{array}{l}\text { LKS } \\
\text { Area } \\
\end{array}$} & \multirow[b]{2}{*}{ stim } & & & \\
\hline & & & & & & & present & \multirow{2}{*}{ Stim } \\
\hline & $x$ & $y$ & $z$ & & & & & \\
\hline Cuneus & -24 & -70 & 17 & BA 18 & VIS & 196 & YES & VIS \\
\hline Fusiform Gyrus & -43 & -64 & -14 & BA 37 & VIS & 2992 & YES & VIS \\
\hline Inferior Occipital Gyrus & -25 & -90 & -3.3 & BA 18 & VIS & 271 & YES & VIS \\
\hline Medial Frontal Gyrus & -3.4 & -6.3 & 53 & BA 6 & AUD & 83 & YES & VIS \\
\hline Middle Occipital Gyrus & -31 & -82 & 6.9 & BA 19 & VIS & 87 & NO & - \\
\hline Precentral Gyrus & -37 & -12 & 41 & BA 6 & VIS & 112 & YES & VIS \\
\hline Precentral Gyrus & -49 & -10 & 46 & BA 4 & AUD & 369 & NO & - \\
\hline Precentral Gyrus & -54 & -6 & 23 & BA 4 & AUD & 117 & YES & VIS \\
\hline Superior Temporal Gyrus & -35 & -35 & 15 & BA 41 & AUD & 132 & YES & AUD \\
\hline Superior Temporal Gyrus & -47 & -25 & 6.9 & BA 41 & AUD & 305 & YES & AUD \\
\hline Superior Temporal Gyrus & -50 & -41 & 16 & BA 13 & AUD & 134 & YES & AUD \\
\hline Superior Temporal Gyrus & -62 & -31 & 8.3 & BA 42 & AUD & 146 & YES & AUD \\
\hline
\end{tabular}

\begin{tabular}{|c|c|c|c|c|c|c|c|c|}
\hline \multirow[b]{2}{*}{ Region } & \multicolumn{5}{|c|}{ Right Hemisphere } & \multirow[b]{2}{*}{ size } & \multicolumn{2}{|c|}{ Controls } \\
\hline & \multicolumn{3}{|c|}{ Center of gravity } & \multirow{2}{*}{ Area } & stim & & present & Stim \\
\hline & $x$ & $y$ & $z$ & & & & & \\
\hline Fusiform Gyrus & 43 & -48 & -17 & BA 37 & VIS & 2232 & YES & VIS \\
\hline Fusiform Gyrus & 28 & -52 & -13 & BA 37 & VIS & 88 & YES & VIS \\
\hline Fusiform Gyrus & 23 & -83 & -19 & BA 19 & VIS & 157 & YES & VIS \\
\hline Fusiform Gyrus & 28 & -74 & -23 & BA 19 & VIS & 563 & NO & - \\
\hline Inferior Occipital Gyrus & 40 & -69 & -7.3 & BA 19 & VIS & 308 & YES & VIS \\
\hline Lingual Gyrus & 27 & -79 & -4.1 & BA 18 & VIS & 1516 & YES & VIS \\
\hline Precentral Gyrus & 47 & -7.5 & 42 & BA 4 & AUD & 330 & YES & VIS \\
\hline Precuneus & 26 & -70 & 21 & BA 31 & VIS & 741 & YES & VIS \\
\hline Precuneus & 24 & -61 & 49 & BA 7 & VIS & 138 & YES & VIS \\
\hline Superior Temporal Gyrus & 49 & -24 & 7.8 & BA 41 & AUD & 2546 & YES & AUD \\
\hline Superior Temporal Gyrus & 41 & -32 & 3.1 & BA 41 & AUD & 208 & NO & - \\
\hline
\end{tabular}
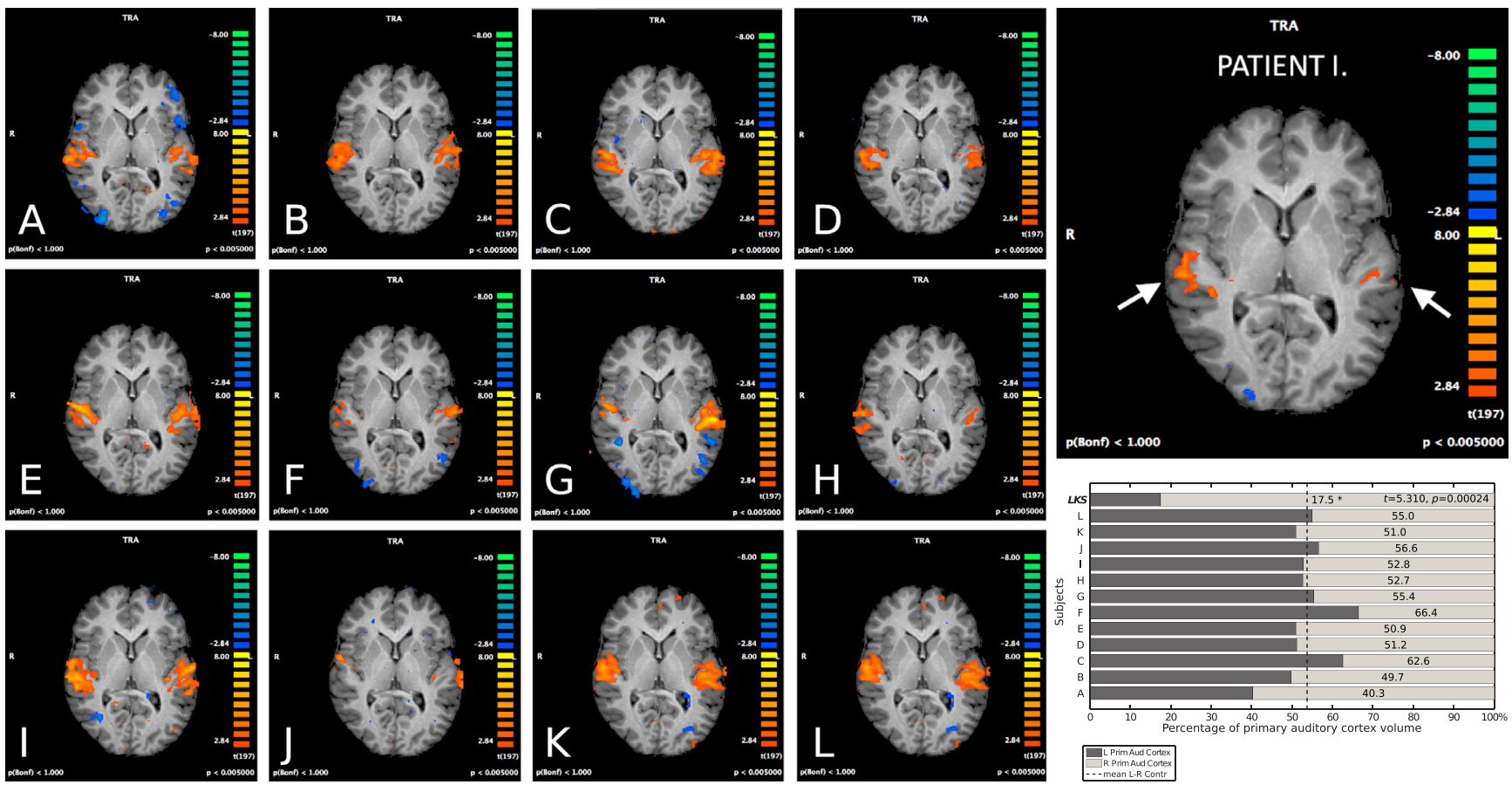

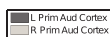

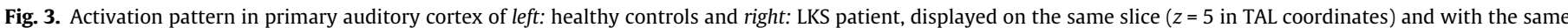

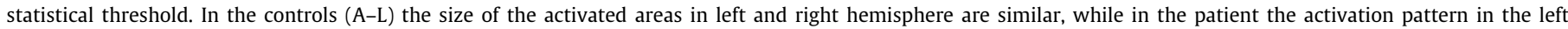

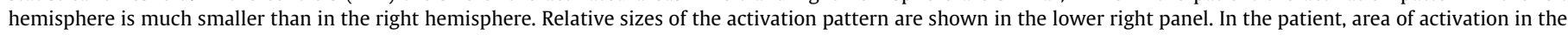
left hemisphere is significantly smaller than in the right hemisphere. 

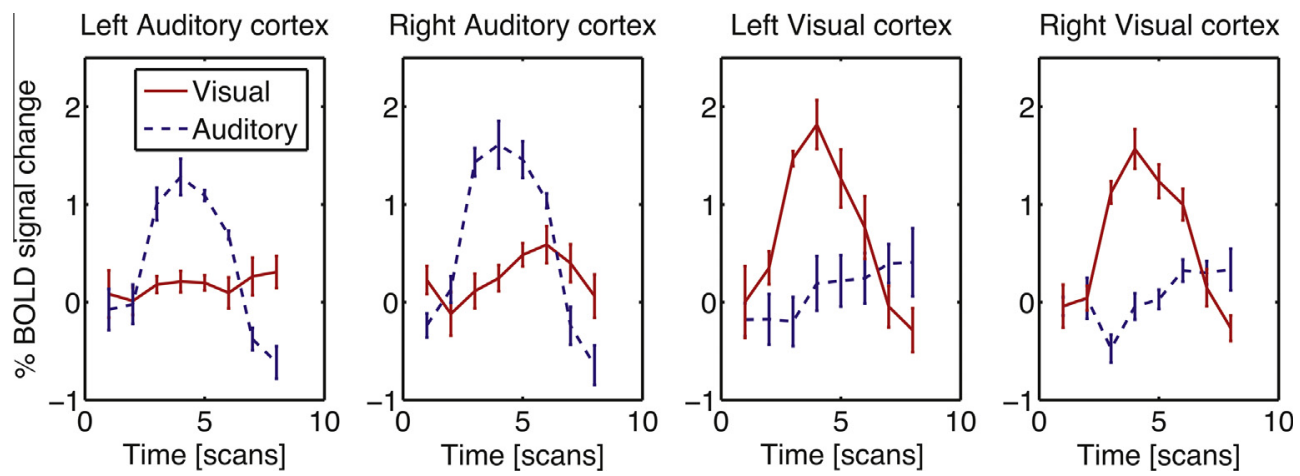

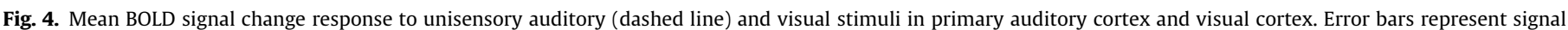

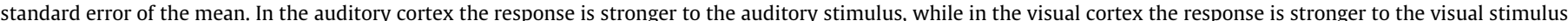
and response to the auditory stimulus stays at baseline level.

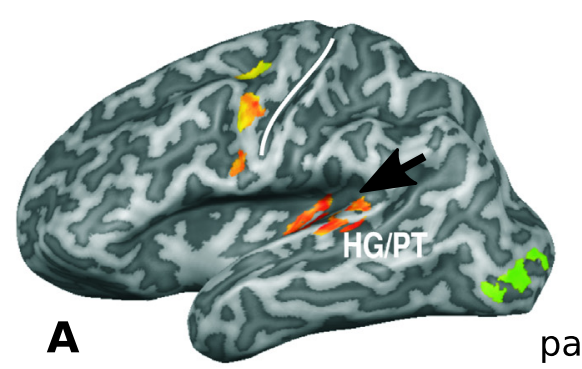

\section{patient}

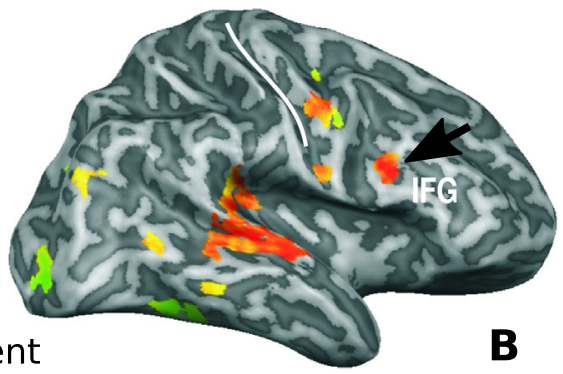

Left hemisphere

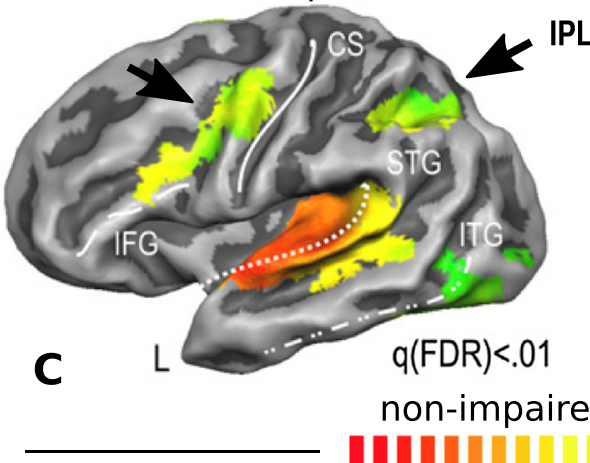

IPL

Right hemisphere

Auditory

Visual

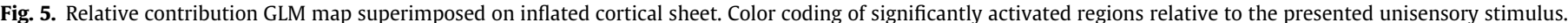

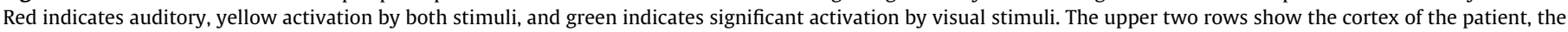

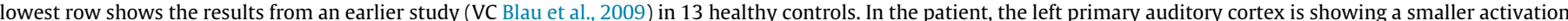

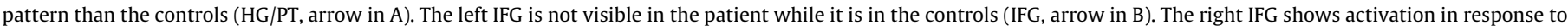

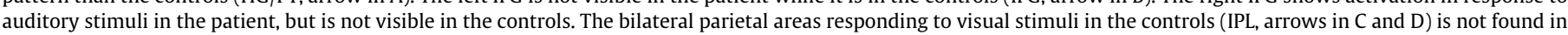

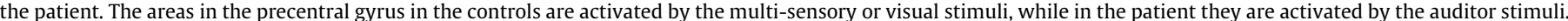

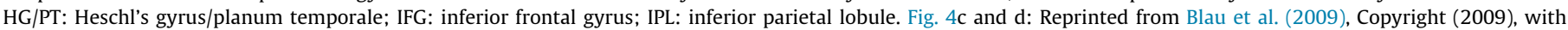
permission from Elsevier. (For interpretation of the references to color in this figure legend, the reader is referred to the web version of this article.)

$R C=\frac{\beta_{v}-\beta_{a}}{\beta_{v}+\beta_{a}}$

The contribution of the auditory stimulus is shown in red, the contribution of the visual stimulus in green and the areas where both stimuli elicit a response are colored yellow. The response pattern to unisensory auditory and visual stimuli is discussed earlier, so the focus is on the regions that are activated by both unisensory stimuli. A detailed description can be found in Table 2. In the right hemisphere, these are superior occipital gyrus (associative visual, BA 19), middle temporal gyrus (BA 21), and superior temporal gyrus STG, as well as cingulate gyrus and a region on the medial part of the superior frontal gyrus (motor planning).
Table 2

Regions significantly activated by contrast congruent $>$ incongruent at $p<0.005$ and a cluster threshold of 5 voxels. $(x, y, z)$ in Talairach coordinates [mm], size in $\mathrm{mm}^{3}$. BA: Brodmann Area; STIM: Stimulus; CON: Congruent; ICON: Incongruent.

\begin{tabular}{lllllll}
\hline Region & $x$ & $y$ & $z$ & Area & Stim & Size \\
\hline $\begin{array}{l}\text { Right hemisphere } \\
\text { Inferior frontal gyrus }\end{array}$ & 8 & 2.1 & 24 & BA 9 & ICON & 219 \\
Middle frontal gyrus & 4 & 28 & 30 & BA 9 & ICON & 151 \\
Left hemisphere & & & & & & \\
Posterior cingulate & -3.5 & -48 & 23 & BA 23 & CON & 190 \\
Middle frontal gyrus & -34 & 43 & 24 & BA 10 & ICON & 144 \\
Insula & -43 & 9.4 & 0.95 & BA 13 & ICON & 203 \\
\hline
\end{tabular}


In the left hemisphere, we also found regions involved in motor planning: medial and middle frontal gyrus, which are activated by both unisensory stimuli.

Results obtained earlier (Blau et al., 2009) in a group of healthy controls are shown in the bottom row of Fig. 5. The 13 controls ( 4 female, mean age 26.8 years, standard deviation 5.4 ) were scanned and analyzed using exactly the same protocol. The patient data shows a smaller activation pattern in response to auditory stimuli in the left primary auditory cortex, indicated by the arrow in Fig. 5a. IFG activation is present in the right hemisphere of the patient (arrow in Fig 5b), but in the left hemisphere of the control group. Furthermore, using this analysis the activation in bilateral superior parietal cortex (black arrows in Fig. 5c and d) of the controls is not found in the patient. The areas in the precentral gyrus in the controls are activated by the multi-sensory or visual stimuli (yellow-green colors, arrows in Fig $5 c$ and d), while in the patients these areas are largely activated by the auditory stimuli.

Since STG is assumed to be involved in integration of letters and sounds (Blau et al., 2009; Van Atteveldt et al., 2004), we investigated the \% BOLD signal change more closely. The \% BOLD signal change was extracted from two ROIs, encompassing left and right STG around previously reported STG coordinates $(-46,-26,6)$, $(45,22,7)$ (Blau et al., 2009). The ROI was defined on the cortical sheet and projected back onto the $2 \mathrm{D}$ slices. The extracted values are not normally distributed, as assessed by the Matlab "normplot" command. Therefore, we used a non-parametric Wilcoxon rank sum test for equal medians to infer on differences between the conditions.

\subsubsection{Letter-sound integration in the superior temporal gyrus}

When looking at the congruent versus the incongruent condition; that is, congruent versus incongruent letter-sound pairs, we find that there is a significant higher response (Wilcoxon rank sum test for equal medians, $(p<1 \mathrm{e}-30)$ for the congruent condition than for the incongruent letter-sound pair in the patient. We compared the fMRI response for the congruent and incongruent condition in the STG with the controls. The mean beta values were extracted from the left and right STG for every subject and for the patient. Fig. 6a shows the distribution of mean beta values in the controls. The beta values of the patient (green $\times$ in Fig. 6a) match those of the controls.

\subsubsection{Multi-sensory interaction}

The Multisensory Interaction index (MSI) is a measure for the amount of integration of the multisensory stimuli relative to the maximum unisensory response
$M S I=\frac{\left(M S-U S_{\max }\right)}{U S_{\max }}$,

where $M S=$ median value of the multisensory stimulus (=congruent or incongruent stimulus), $U S_{\max }=$ median of the largest unisensory response. A positive $M S I$ value indicates a response enhancement with respect to the unisensory stimulus, while a negative MSI represents suppression of the response.

In Fig. 6b, the MSI in the left and right STG (same ROI as used for Fig. 6a) for this subject is shown. Bilaterally, the MSI is more negative for the incongruent as for the congruent condition (L: -0.167 versus -0.479 ; $\mathrm{R}$ : -0.173 versus -0.465 ), indicating a modulation of the response, which possibly represents integration of letters and sounds.

\subsection{DTI based fiber tracking}

Full brain fractional anisotropy (Fig. S1) and mean diffusivity maps (Fig. S2) are available as supplemental materials.

\subsubsection{Fiber reconstructions from Heschl's gyrus}

The reconstructed fiber tracts originating from left and right HG are shown in Fig. 7a. From the left HG reconstructed fibers curve upwards around the sylvian fissure and project into the superior bank of the sylvian fissure and inferior frontal gyrus. A small portion of the fibers projects anteriorly into the tip of the temporal lobe, and posterior segment projects into the occipital lobe.

In the right hemisphere a proportion of the reconstructed fibers curve around the posterior part of the sylvian fissure. A segment projects into superior parietal cortex, while another segment projects forwards medial to the sylvian fissure into the inferior frontal lobe. A third segment projects into the cerebellum.

\subsubsection{Arcuate fasciculus fiber reconstructions}

Fiber reconstructions of the arcuate fasciculus in both hemispheres are shown in Fig. 7b and c. The left hemisphere AF reconstruction in Fig. $7 \mathrm{~b}$ runs from the posterior parts of the superior and middle temporal gyri, then arches around the anterior part of the sylvian fissure and ends posterior of the inferior frontal gyrus.

The right hemisphere's AF main segment (blue in Fig. 7c) follows a similar path as in the left hemisphere, from the superior MTG/STG around the posterior part of the sylvian fissure to parts posterior of the inferior frontal gyrus. Two other segments were found in the tractography results; one posterior segment (green in Fig. 7c) running from superior MTG/STG to inferior parietal lobe,
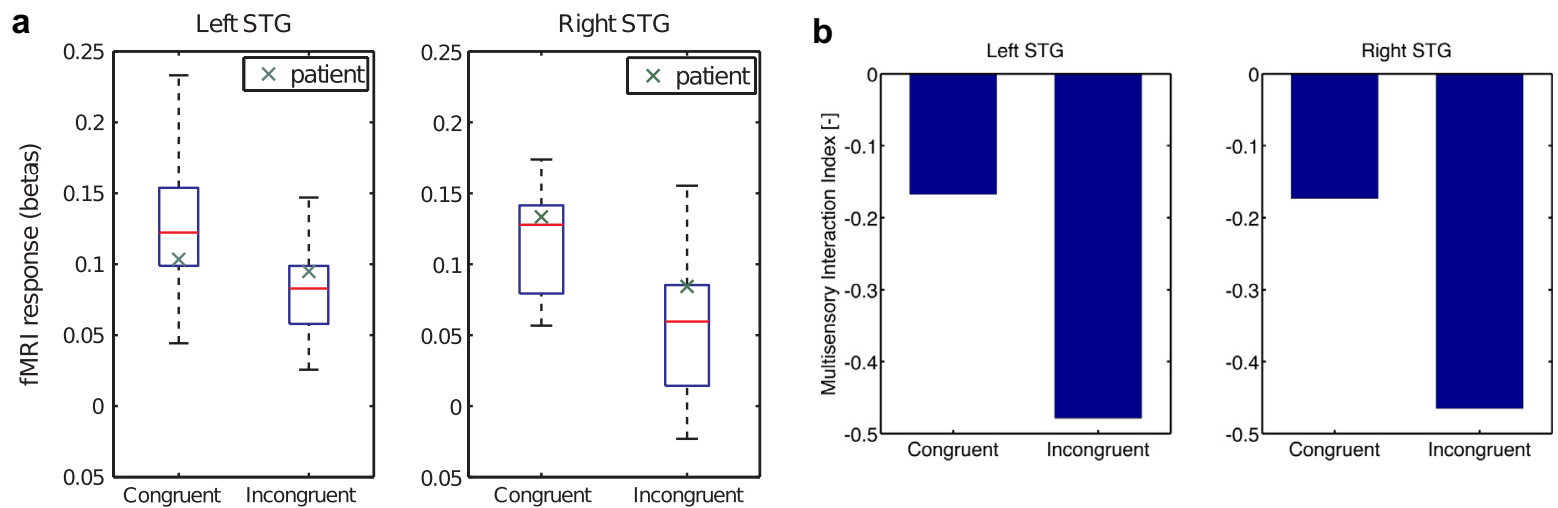

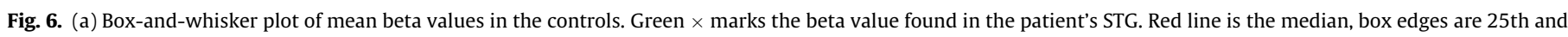

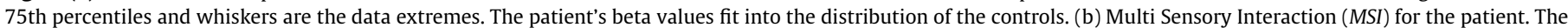

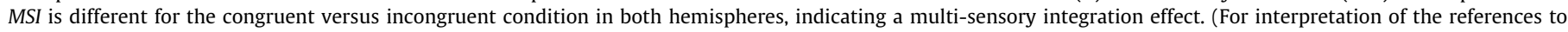
color in this figure legend, the reader is referred to the web version of this article.) 


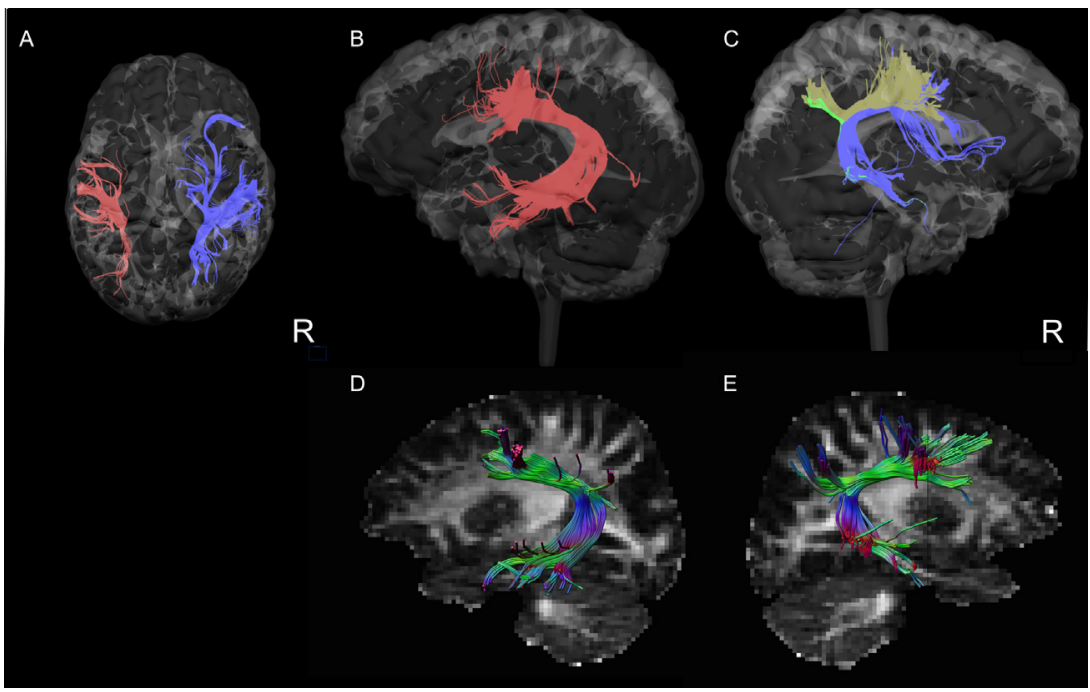

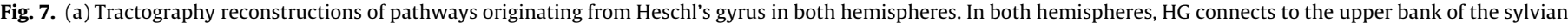

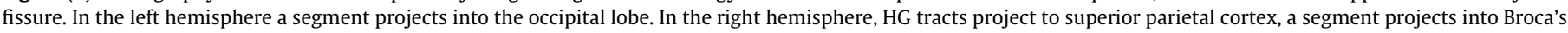

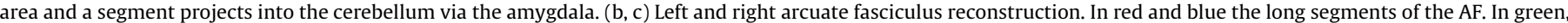

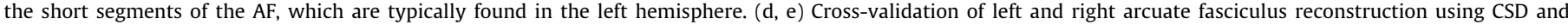

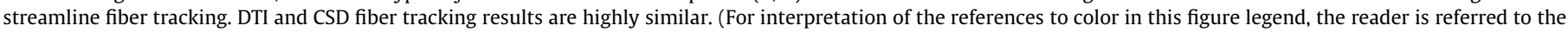
web version of this article.)
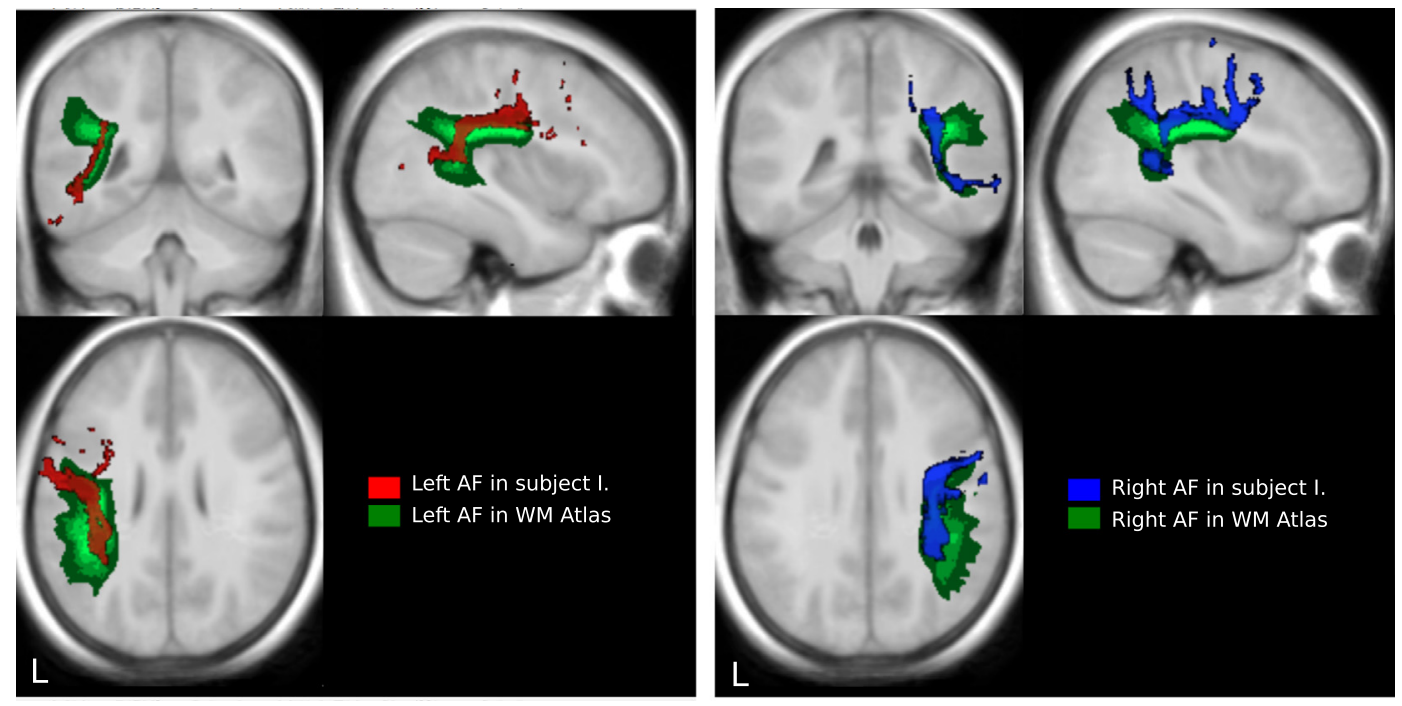

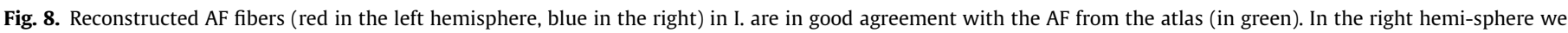

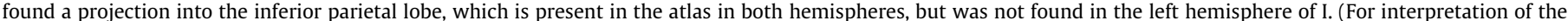
references to color in this figure legend, the reader is referred to the web version of this article.)

and a segment running from inferior parietal lobe toward parts posterior of the inferior frontal gyrus, which is the yellow segment in Fig. 7c.

The lateralization index, computed for the number of streamlines in the AF is (\#streamlines right - \#streamlines left)/(right + left $)=(17,641-12,470) / 30,111=0.172$, which means the AF is right lateralized. In healthy volunteers, the $\mathrm{AF}$ is not lateralized (Thiebaut de Schotten et al., 2011).

4.2.3. Comparison of the arcuate fasciculus to the white matter atlas

In Fig. 8 the overlap of the left and right AF in I. with the WM atlas AF is shown. The reconstructed fibers in I. are in good agreement with the atlas. In the left hemisphere however, the segment connecting the temporal and inferior parietal lobe is not found in I. while it is present in the WM atlas of control subjects. In the right hemisphere, $\mathrm{AF}$ reconstruction is similar to the controls and we did find a segment connecting inferior parietal lobe with STG/MTG.

\section{Discussion}

\subsection{Right lateralized response to auditory speech sounds}

In healthy adults investigated with the same experiment, the response to unisensory speech sounds is bilaterally similar and spread out over the PT/HG (Van Atteveldt et al., 2004). In our patient, we observed a larger response area in the right compared to the left hemisphere. In the right hemisphere a coherent area of activation $\left(2754 \mathrm{~mm}^{3}\right)$ was found compared to a more scattered and in total smaller area of activation $\left(583 \mathrm{~mm}^{3}\right)$ in the left hemisphere. The deviating response in the left hemisphere cannot be 
related to a hearing deficit, since the audiogram is normal and the BOLD response in the primary auditory cortex is comparable to that in healthy subjects studied with the same experiment (Van Atteveldt et al., 2004). A previous single case fMRI experiment, evaluating the response to spoken words in a left-handed post-epileptic phase LKS patient (Sieratzki, Calvert, Brammer, David, \& Woll, 2001) revealed a similar, although less pronounced, right lateralization: right auditory cortex showed an increase response relative to the left hemisphere, despite the fact that the epileptic foci were on the right side in the reported case. In a recent single case fMRI study of a LKS patient during the active epileptic phase, a right lateralization of language functions was also observed (Datta et al., 2013).

Although the activated cortical area differs greatly in the right and the left hemisphere, the shape of BOLD responses to the unisensory stimuli in auditory and visual cortex are in line with results obtained earlier in healthy subjects (Blau et al., 2009), indicating that early auditory and visual cortex function normally and are able to relay information up to higher cortical areas for further processing. Since the subject is proficient in reading, the processing of visual letters is therefore considered to be effective.

\subsection{Pre-central gyrus and pre-SMA activation in response to auditory speech sounds}

Activation of pre-motor areas has been reported for speech sound perception (Wilson, Pinar Saygin, Sereno, \& Iacoboni, 2004). These regions are related to speech production using the mouth and lips and have been found to be robustly activated in healthy subjects while passively listening to meaningless monosyllables (Wilson et al., 2004). The reported MNI coordinates from left precentral gyrus $(-50,-6,47)$ and right precentral gyrus $(55,-3,45)$ correspond to TAL coordinates: left $(x, y, z)=(-49.5,-3.7,43.5)$, right $(54.5,-1,41.5)$ and overlap the premotor areas in this subject, which are centered around $(-49,-10,46)$ and $(47,-7.5,42)$. A surprising finding is that in healthy subjects, similar areas are more strongly activated by the visual letter stimuli than by the auditory stimuli.

Considering the fact that finger spelling is the native language of this subject, it is surprising that the pre-motor areas for finger/hand movements are not recruited in this experiment. The motor areas related to finger movements lie a bit more superior $(-35.5,-14.6,65.3)$, as reported in Moore et al. (2000). Finger twitching is observed frequently in LKS patients, who use sign language/finger spelling, when listening to spoken language (Sieratzki et al., 2001) and personal observations with multiple patients (author WP). It could therefore very well be that for more complex words and sentences, rather than the single letters presented in the current study, finger spelling or sign language is adopted as an aid to understanding.

\subsection{Integration of letter-sound pairs in the STG}

In healthy subjects, auditory cortex and auditory association areas in the superior temporal cortex (STS/STG) have been implicated in the integration of letters and speech sounds. Greater neural responses were observed when the letters and the sounds were matching (congruent) as opposed to mismatching (incongruent), indicating specialized processing for the learned audiovisual association between letters and sounds. In contrast, dyslexic adults and children have been shown to be impaired in their integration of letters and speech sounds in those same temporal brain regions (Blau et al., 2010, 2009).

In our patient, the observed activity in the STG was similar to that of healthy subjects. When looking at the MSI index, we observed a bilateral modulation of the BOLD response between congruent and incongruent condition, which has been interpreted as indication for multi-sensory integration (Blau et al., 2009; Van Atteveldt et al., 2004). For our LKS patient, it might indicate that processing of single letters is functionally intact, and that problems occur at a higher order information processing of words or sentences.

\subsection{DTI findings}

In this study, we investigated the white matter pathways originating from Heschl's gyrus as well as the main white matter structure relevant for language processing, the arcuate fasciculus, with DTI-based fiber tracking. We observed significant differences between LKS patient and healthy controls. Fibers originating from Heschl's gyrus seem to be left-right inverted with respect to HG fibers found in a control study (Barrick, Lawes, \& Clark, 2004). In addition, whereas the long segment of the arcuate fasciculus was as in healthy controls (visible in both hemispheres), wee did not find the short segments in the left arcuate fibers reported in healthy subjects (Catani et al., 2007; Thiebaut de Schotten et al., 2011). In contrast the short segments seemed to be intact in the right hemisphere.

Data on the white matter pathways originating from HG investigated with DW-MRI is sparse. One study found a posterior pathway between posterior regions and Heschl's gyrus (HG) and posterior Superior Temporal Gyrus (STG) using DTI based probabilistic fiber tracking (Upadhyay et al., 2007). An anterior pathway, connecting anterior HG and anterior STG, was also found, but this pathway was less dense than the anterior pathway (Upadhyay et al., 2008). Another study using DTI based deterministic fiber tracking reported asymmetrical pathways (Barrick et al., 2004 ) in 30 healthy subjects. The study reported that in the left hemisphere reconstructed fibers pass beneath the planum temporale and terminated within the insular cortex and inferior frontal gyrus. In the right hemisphere, fibers passed beneath planum temporale and terminated within parietal and occipital lobes. Evidence from functional MRI showed that HG is connected to anterior STG, STS and IFG in the left hemisphere (Scott \& Johnsrude, 2003), which seem to complement the white matter connection pattern.

In the patient, portions of the fibers originating from HG project into anterior parts of the STG in both hemispheres, as well as additional fibers connecting HG in the left hemisphere with the pole of the temporal lobe and the occipital lobe. In the right hemisphere, we found additional fibers projecting toward inferior parietal and inferior frontal lobe, which seemed to be integrated within the arcuate fasciculus to some degree. Fibers descending into the cerebellum as part of the auditory processing pathway were also found. Interestingly, in the patient, the fiber tracts originating from right HG projecting to inferior frontal areas seemed to resemble the left inferior frontal fibers found in healthy controls (Barrick et al., 2004).

If we look at the arcuate fasciculus, it was shown in a study with 40 healthy subjects (Catani et al., 2007) in all subjects that STG/STS was connected to IFG via the long segment of the arcuate fasciculus (AF) in the left hemisphere. In the right hemisphere, the majority $(62.5 \%)$ of subjects did not have a homologue AF connection. In $20 \%$ of the subjects, the right AF was found to be weaker, and in $17.5 \%$ of subjects it was equal to the left AF. This indicates a leftward lateralization of the arcuate fasciculus. Another study (Gharabaghi et al., 2009) reported the presence of a right AF homologue in 12/12 subjects, but did not report a lateralization index, since only the right AF was investigated. A very recent study in 40 healthy subjects (Thiebaut de Schotten et al., 2011) reported again a strong leftward lateralization of the AF long segment. Our results show the presence of the long segment of the AF in both 
hemispheres. Additional to the long segment of the AF connecting superior STS and STG to IFG in the left hemisphere, there are two shorter segments reported to connect superior STS and STG to the inferior parietal lobule (associative auditory cortex) and connecting the inferior parietal lobule to IFG (Catani et al., 2007; Thiebaut de Schotten et al., 2011). The shorter segments of the AF were also found in the right hemisphere in healthy subjects (Catani et al., 2007; Gharabaghi et al., 2009; Thiebaut de Schotten et al., 2011). We did not find evidence for these short segments of AF in the left hemisphere, while these segments are consistently found in the left hemisphere of healthy subjects, as also reported in the WM atlas. In the current study, the short segments projecting to the inferior parietal lobe were found to exist only in the right hemisphere.

It should be noted that differences between the patient data and results from the literature might also exist because of methodological differences, e.g. different acquisition and processing of the DTI data. It is difficult to estimate the impact of methodological differences in across-subjects comparisons. Most recently, first suggestions have been made how to quantify differences between tractography methods when applied to global connectivity measures (Bastiani, Shah, Goebel, \& Roebroeck, 2012). However, it remains a challenge to estimate the effect of the tractography method in local fiber bundles as we have tracked here. Given the high number of subjects in the atlas we believe it is a reliable source for comparison.

The current study is limited in the sense that fiber tracts from an individual subject are investigated. To our knowledge, post-mortem data from LKS patients is not available and clearly we cannot resort to animal studies. Therefore, we need to rely on white matter atlases obtained in healthy subjects. A dissimilarity in fiber tracts found here and the atlas might also arise from differences in the regions of interest used to seed the fibers. The data in the atlas used here (Thiebaut de Schotten et al., 2011) was obtained on a $1.5 \mathrm{~T}$ system with a $b$-value of $1300 \mathrm{~s} / \mathrm{mm}^{2}$, and was processed with different software and different fiber tracking stopping criteria $(F A=0.2$, angle $=45$ degrees $)$, which might influence the comparison. We have cross-validated our AF fiber tracking results obtained with DTI and streamline fiber tracking with a CSD reconstruction of the data combined with streamline fiber tracking. The CSD method is better able to resolve kissing and crossing fiber tracts. Although our data is not particularly suited for CSD reconstruction because a higher $b$-value up to $b=3000 \mathrm{~s} / \mathrm{mm}^{2}$ is recommended for CSD (Tournier, Calamante, Gadian, \& Connelly, 2004), we have obtained highly similar results between DTI and CSD fiber tracking.

Within the healthy system, the short segments in the left hemisphere are possibly connecting the arcuate fasciculus with a section on the border of the left inferior parietal lobe and sylvian fissure, termed the sylvian-parietal temporal area (Spt) (Hickok, 2009; Hickok \& Poeppel, 2007). Spt is regarded crucial for sensory-motor integration of speech or non- speech sounds (e.g. music). Spt is also crucial with regard to verbal short term memory functions, as it is part of phonological short-term memory circuit. Consequently, this area is essential when learning to speak, online guidance of speech sequences, and for acquiring new vocabulary (Hickok \& Poeppel, 2007).

LKS patients in the active and recovered phase suffer from permanent dysfunction of associative auditory cortex as measured in a dichotic listening task during EEG recordings (Metz-Lutz et al., 1999; Plaza et al., 2001; Wioland et al., 2001). Furthermore, short-term phonological memory is consistently found to be impaired in LKS, even in patients who have recovered fairly well (Majerus et al., 2003). It was found to be weak in the patient in the early active stage of LKS (Pullens, 1995). In addition, she currently continues to make mistakes in sentence construction, word form, melody and accent and has troubles with learning new vocabulary when presented verbally (observations by WP) Our finding indicate a potential correlation of missing short fasciculate segments in the left hemisphere with the observed language deficits.

The results presented here in fibers originating from Heschl's gyrus and the arcuate fasciculus suggest a rightward lateralization of language-related WM pathways. Currently we can only speculate about the re-organization of these fibers in the right hemisphere. There is some evidence that in deaf signers, the right hemisphere is more involved in sign language processing than the left hemisphere (Neville et al., 1998) but this is being debated, see (MacSweeney, Capek, Campbell, \& Woll, 2008) for an overview. Since the patient's "native language" is sign language, the right hemisphere has possibly taken over language processing functions from the left hemisphere. A second possibility is that the left temporal lobe was more severely affected during the epileptic phase, causing the right hemisphere to take over some functions.

Based on the models described in the introduction and the fMRI and DTI data presented here we have constructed a hypothetical model of language processing in LKS. Key observations are that the right auditory cortex is less activated, that the right IFG is activated instead of the left IFG in healthy controls, that IPL activation is absent in the patient data, and that the auditory stimulus activates pre-motor areas instead of the visual stimulus in control subjects.

We hypothesize that the absence of arcuate fibers directed toward the left inferior parietal lobe causes auditory information to not interact with the motor system, thereby restricting phonological short-term memory, (speech) sound-motor interaction and online feedback of speech and might be one of the neuronal factors underlying LKS.

Combined with previous findings that short-term phonological memory is weak in LKS (Majerus et al., 2003), we suggest that the IPL and auditory sensory-motor integration is disrupted in LKS. Moreover, visual language (lip reading or finger spelling) has developed into the more natural language in this patient. As a consequence, when an auditory stimulus is presented, this requires a higher workload for the pre-motor areas translating the auditory input into a visual representation (i.e. a word form of the mouth or a character from a finger spelling alphabet) instead of the other way around as in healthy subjects, where a visual input seems to be translated into a motor representation.

\subsection{Conclusions and Hypothesis}

In this study, the integration of visual letters and auditory sounds in a recovered, post-epileptic phase Landau-Kleffner patient was investigated with fMRI. To our knowledge this is one of the first fMRI studies investigating the underlying functional and neuro-anatomical mechanisms of LKS.

With DTI-based fiber tracking the neuro-anatomy of the language system was investigated. We observed a lack of fibers projecting from the arcuate fasciculus to the left inferior parietal lobe, which is possibly related to the disruption in short-term phonological memory and auditory sensory-motor integration in LKS.

The results from the letter-sound integration in the STG suggest that in recovered LKS, language processing of single letters is probably organized in the same way as in normals, suggesting the language system is still intact within local STG circuits. However, the information might not be accessible in the same way as in normal adults related to a disruption of the auditory sensory-motor integration and short term phonological memory in the IPL.

Although this article is based on fMRI data of a single subject, the outcome seems to be in line with observations and data from researchers in the field of LKS. Until now there was no evidence, 
but anecdotal, over the use of visually supported communication systems in the treatment of this syndrome. Further investigation on al larger scale, including more subjects and a lager test battery will give us more clarity over the importance of language acquisition through visual information channels.

\section{Appendix A. Supplementary material}

Supplementary data associated with this article can be found, in the online version, at http://dx.doi.org/10.1016/j.bandc.2015.07. 003.

\section{References}

Appleton, R. (1995). The Landau-Kleffner syndrome. Archives of Disease in Childhood, 72(5), 386-387.

Barrick, T., Lawes, I., \& Clark, C. (2004). White matter pathway asymmetry corresponds to auditory spatial and language lateralisation. In Proc ISMRM (Vol. 11, p. 334).

Bastiani, M., Shah, N. J., Goebel, R., \& Roebroeck, A. (2012). Human cortical connectome reconstruction from diffusion weighted MRI: The effect of tractography algorithm. NeuroImage, 62(3), 1732-1749. http://dx.doi.org/ 10.1016/j.neuroimage.2012.06.002.

Blau, V., Reithler, J., Van Atteveldt, N., Seitz, J., Gerretsen, P., Goebel, R., et al. (2010). Deviant processing of letters and speech sounds as proximate cause of reading failure: A functional magnetic resonance imaging study of dyslexic children. Brain, 133(Pt 3), 868-879.

Blau, V., Van Atteveldt, N., Ekkebus, M., Goebel, R., \& Blomert, L. (2009). Reduced neural integration of letters and speech sounds links phonological and reading deficits in adult dyslexia. Current Biology, 19, 503-508.

Catani, M., Allin, M., Husain, M., Pugliese, L., Mesulam, M., Murray, R., et al. (2007), Symmetries in human brain language pathways correlate with verbal recall. PNAS, 43(23), 17163-17168.

Catani, M., Jones, D., \& fFytche, D. (2005). Perisylvian language networks of the human brain. Annals of Neurology, 57(1), 8-16. http://dx.doi.org/10.1002/ ana.20319.

Cavina-Pratesi, C., Kentridge, R. W., Heywood, C. A., \& Milner, A. D. (2009). Separate processing of texture and form in the ventral stream: Evidence from fMRI and visual agnosia. Cerebral Cortex, 20(2), 433-446. http://dx.doi.org/10.1093/ cercor/bhp111.

Cockerell, I., Bølling, G., \& Nakken, K. O. (2011). Landau-Kleffner syndrome in Norway: Long-term prognosis and experiences with the health services and educational systems. Epilepsy $\mathcal{E}$ Behavior: EEB, 21(2), 153-159. http:// dx.doi.org/10.1016/j.yebeh.2011.03.019.

Datta, A. N., Oser, N., Ramelli, G. P., Gobbin, N. Z., Lantz, G., Penner, I. K., et al. (2013). BECTS evolving to Landau-Kleffner syndrome and back by subsequent recovery: A longitudinal language reorganization case study using fMRI, source EEG, and neuropsychological testing. Epilepsy and Behavior, 27(1), 107-114. http://dx.doi.org/10.1016/j.yebeh.2012.12.025.

De Gelder, B., \& Vroomen, J. (2000). The perception of emotions by ear and by eye. Cognition \& Emotion, 14, 289-311. http://dx.doi.org/10.1080/ 026999300378824.

Denes, G. (1998). Landau-Kleffner syndrome; Clinical and linguistic aspects. In B. Stemmer \& H. Whitacker (Eds.), Handbook of neurolinguistics. San Diego: Academic Press.

Deonna, T., Prelaz-Girod, A., Mayor-Dubois, C., \& Roulet-Perez, E. (2009). Sign language in Landau-Kleffner syndrome. Epilepsia, 50(suppl 7), 77-82.

Fandiño, M., Connolly, M., Usher, L., Palm, S., \& Kozak, F. (2011). Landau-Kleffner syndrome: A rare auditory processing disorder series of cases and review of the literature. International Journal of Pediatric Otorhinolaryngology, 75, 33-38.

Feekery, C., Parryfielder, B., \& Hopkins, I. (1993). Landau-Kleffner syndrome - 6 Patients including discordant monozygotic twins. Pediatric Neurology, 9(1), 49-53.

Gharabaghi, A., Kunath, F., Erb, M., Saur, R., Heckl, S., Tatagiba, M., et al. (2009). Perisylvian white matter connectivity in the human right hemisphere. BMC Neuroscience, 6, 10-15. http://dx.doi.org/10.1186/1471-2202-10-15.

Goebel, R., Esposito, F., \& Formisano, E. (2006). Analysis of functional image analysis contest (FIAC) data with Brainvoyager QX: From single-subject to cortically aligned group general linear model analysis and self-organizing group independent component analysis. Human Brain Mapping, 27, 392-401.

Hickok, G. (2009). The functional neuroanatomy of language. Physics of Life Reviews, 6(3), 121-143. http://dx.doi.org/10.1016/j.plrev.2009.06.001.

Hickok, G., Houde, J., \& Rong, F. (2011). Sensorimotor integration in speech processing: Computational basis and neural organization. Neuron, 69, 407-422. http://dx.doi.org/10.1016/j.neuron.2011.01.019.

Hickok, G., \& Poeppel, D. (2007). The cortical organization of speech processing. Nature Reviews Neuroscience, 8, 393-402.

Hirsch, E., Paola, M., Rudolf, G., Seegmuller, C., De Saint, A., Maquet, P., et al. (2006). Landau-Kleffner syndrome is not an eponymic badge of ignorance. Epilepsy Research, 239-247. http://dx.doi.org/10.1016/j.eplepsyres.2006.02.010.

Kaufman, A., \& Kaufman, N. (1985). Kaufman test of educational achievement: Comprehensive form manual. Circle Pines, MN: American Guidance Service.
Landau, W., \& Kleffner, F. (1957). Syndrome of acquired aphasia with convulsive disorder in children. Neurology, 7, 523-530.

Lanzi, G., Veggiotti, P., Conte, S., Partesana, E., \& Resi, C. (1994). A correlated fluctuation of language and EEG abnormalities in a case of the Landau-Kleffner syndrome. Brain \&' Development, 16(4), 329-334.

Leemans, A., Jeurissen, B., Sijbers, J., \& Jones, D. (2009). ExploreDTI: A graphical toolbox for processing, analyzing, and visualizing diffusion MR data. In Proc ISMRM (p. 3537).

Lieberman, M., \& Cunningham, W. (2009). Type I and Type II error concerns in fMRI research: Re-balancing the scale. SCAN, 4, 423-428.

MacSweeney, M., Capek, C., Campbell, R., \& Woll, B. (2008). The signing brain: The neurobiology of sign language. Trends in Cognitive Sciences, 12, 432-440.

Majerus, S., Laureys, S., Collette, F., Del Fiore, G., Degueldre, C., Luxen, A., Van Der Linden, M., Maquet, P., \& Metz-Lutz, M. N. (2003). Phonological short-term memory networks following recovery from Landau and Kleffner syndrome. Human Brain Mapping, 19, 133-144.

Metz-Lutz, M., de Saint Martin, A., Hirsch, E., Maquet, P., \& Marescaux, C. (1999). Impairment in auditory verbal processing and dichotic listening after recovery of epilepsy in Landau and Kleffner syndrome. Brain and Cognition, 40(1), 193-197.

Moore, C., Stern, C., Corkin, S., Fischl, B., Gray, A., Rosen, B., et al. (2000). Segregation of somatosensory activation in the human rolandic cortex using fMRI. Journal of Neurophysiology, 84, 558-569.

Mori, S., \& Van Zijl, P. C. (2002). Fiber tracking: Principles and strategies - A technical review. NMR in Biomedicine, 15, 468-480.

Mulder, J., Dekker, R., \& Dekker, P. (1993). Kaufman intelligence test-Dutch Edition. Leiden, NL: PITS,

Neville, H. J., Bavelier, D., Corina, D., Rauschecker, J., Karni, A., Lalwani, A., et al. (1998). Cerebral organization for language in deaf and hearing subjects: Biological constraints and effects of experience. Proceedings of the National Academy of Sciences of the United States of America, 95(3), 922-929. <http:// www.pubmedcentral.nih.gov/articlerender.fcgi ?artid=33817\&tool= pmcentrez\&rendertype $=$ abstract $>$.

Pedro, V., \& Leisman, G. (2005). Hemispheric integrative therapy in Landau-Kleffner syndrome: Applications for rehabilitation sciences. International Journal of Neuroscience, 115(8), 1227-1238.

Perez, E. R., Davidoff, V., Prelaz, A. C., Morel, B., Rickli, F., Metz-Lutz, M. N., et al. (2001). Sign language in childhood epileptic aphasia (Landau-Kleffner syndrome). Developmental Medicine and Child Neurology, 43(11), 739-744.

Plaza, M., Rigoard, M., Chevrie-Muller, C., Cohen, H., \& Picard, A. (2001). Short-term memory impairment and unilateral dichotic listening extinction in a child with Landau-Kleffner syndrome: Auditory or phonological disorder? Brain and Cognition, 46(1-2), 235-240.

Pullens, W. (1995). [Landau-Kleffner syndrome; The effect of fingerspelling on language processing] (in Dutch).

Scott, S., \& Johnsrude, I. (2003). The neuroanatomical and functional organization of speech perception. Trends in Neurosciences, 26, 100-107.

Shiraishi, H., Takano, K., Shiga, T., Okajima, M., \& Sudo, A. (2007). Possible involvement of the tip of temporal lobe in Landau-Kleffner syndrome. Brain and Development, 29, 529-533. http://dx.doi.org/10.1016/j.braindev.2007. 01.011.

Sieratzki, J., Calvert, G., Brammer, M., David, A., \& Woll, B. (2001). Accessibility of spoken, written, and sign language in Landau-Kleffner syndrome: A linguistic and functional MRI study. Epileptic Disorders, 3(2), 79-89.

Sigalovsky, I., Fischl, B., \& Melcher, J. (2006). Mapping an intrinsic MR property of gray matter in auditory cortex of living humans: A possible marker for primary cortex and hemispheric differences. Neurolmage, 32, 1524-1537. http:// dx.doi.org/10.1016/j.neuroimage.2006.05.023.

Smith, S. M., Jenkinson, M., Woolrich, M. W., Beckmann, C. F., Behrens, T. E. J., Johansen-Berg, H., et al. (2004). Advances in functional and structural MR image analysis and implementation as FSL. NeuroImage, 23(S1), 208-219.

Stefanatos, G. (2011). Changing perspectives on Landau-Kleffner syndrome. The Clinical Neuropsychologist, 25(6), 963-988. http://dx.doi.org/10.1080/13854046. 2011.614779.

Steinlein, O. (2009). Epilepsy - aphasia syndromes. Expert Review of Neurotherapeutics, 9(6), 825-833.

Takeoka, M., Jr., Riviello, J. J., Duffy, F. H., Kim, F., Kennedy, D. N., Holmes, G. L., et al (2004). Bilateral volume reduction of the superior temporal areas in LandauKleffner syndrome. Neurology, 63, 1289-1292.

Thiebaut de Schotten, M., Ffytche, D. H., Bizzi, A., Dell'Acqua, F., Allin, M., Walshe, M., et al. (2011). Atlasing location, asymmetry and inter-subject variability of white matter tracts in the human brain with MR diffusion tractography. NeuroImage, 54(1), 49-59. http://dx.doi.org/10.1016/j.neuroimage.2010.07.055.

Tournier, J. D., Calamante, F., Gadian, D. G., \& Connelly, A. (2004). Direct estimation of the fiber orientation density function from diffusion-weighted MRI data using spherical deconvolution. Neuroimage, 23, 1176-1185.

Upadhyay, J., Ducros, M., Knaus, T. A., Lindgren, K. A., Silver, A., Tager-Flusberg, H., et al. (2007). Function and connectivity in human primary auditory cortex: A combined fMRI and DTI study at 3 Tesla. Cerebral Cortex (New York, N.Y.: 1991), 17(10), 2420-2432. http://dx.doi.org/10.1093/cercor/bhl150.

Upadhyay, J., Silver, A., Knaus, T., Lindgren, K., Ducros, M., Kim, D.-S., et al. (2008) Effective and structural connectivity in the human auditory cortex. The Journal of Neuroscience, 28, 3341-3349. http://dx.doi.org/10.1523/JNEUROSCI.443407.2008 .

Van Atteveldt, N., Formisano, E., Goebel, R., \& Blomert, L. (2004). Integration of letters and speech sounds in the human brain. Neuron, 43, 271-282. 
Van Atteveldt, N., Roebroeck, A., \& Goebel, R. (2009). Interaction of speech and script in human auditory cortex: Insights from neuro-imaging and effective connectivity. Hearing Research, 258(1-2), 152-164. http://dx.doi.org/10.1016/ j.heares.2009.05.007.

Van den Stock, J., Grèzes, J., \& de Gelder, B. (2008). Human and animal sounds influence recognition of body language. Brain Research, 1242, 185-190. http:// dx.doi.org/10.1016/j.brainres.2008.05.040.

Wilson, S., Pinar Saygin, A., Sereno, M., \& Iacoboni, M. (2004). Listening to speech activates motor areas involved in speech production. Nature Neuroscience, 7(7), $701-702$
Wioland, N., Rudolf, G., \& Metz-lutz, M. N. (2001). Electrophysiological evidence of persisting unilateral auditory cortex dysfunction in the late outcome of Landau and Kleffner syndrome. Clinical Neurophysiology, 112, 319-323.

Zhuang, J., Hrabe, J., Kangarlu, A., Xu, D., Bansal, R., Branch, C., et al. (2006). Correction of eddy-current distortions in diffusion tensor images using the known directions and strengths of diffusion gradients. Journal of Magnetic Resonance Imaging, 1193, 1188-1193. http://dx.doi.org/10.1002/jmri.20727.

Zivi, A., Broussaud, G., Daymas, S., Hazard, J., \& Sicard, C. (1990). Epilepsia-acquired aphasia syndrome with psychosis - Report of a case. Annales De Pediatrie, 37(6), 391-394. 